\title{
DENOMINATORS OF BERNOULLI POLYNOMIALS
}

\author{
OLIVIER BORDELLÈS, FLORIAN LUCA, PIETER MOREE, \\ AND IGOR E. SHPARLINSKI
}

Abstract. For a positive integer $n$ let

$$
\mathfrak{P}_{n}=\prod_{\substack{p \\ s_{p}(n) \geqslant p}} p
$$

where $p$ runs over primes and $s_{p}(n)$ is the sum of the base $p$ digits of $n$. For all $n$ we prove that $\mathfrak{P}_{n}$ is divisible by all "small" primes with at most one exception. We also show that $\mathfrak{P}_{n}$ is large, has many prime factors exceeding $\sqrt{n}$, with the largest one exceeding $n^{20 / 37}$. We establish Kellner's conjecture, which says that the number of prime factors exceeding $\sqrt{n}$ grows asymptotically as $\kappa \sqrt{n} / \log n$ for some constant $\kappa$ with $\kappa=2$. Further, we compare the sizes of $\mathfrak{P}_{n}$ and $\mathfrak{P}_{n+1}$, leading to the somewhat surprising conclusion that although $\mathfrak{P}_{n}$ tends to infinity with $n$, the inequality $\mathfrak{P}_{n}>\mathfrak{P}_{n+1}$ is more frequent than its reverse.

\section{INTRODUCTION}

1.1. Motivation. For positive integers $n$ and $b \geqslant 2$ let $s_{b}(n)$ be the sum of the base $b$-digits of $n$. The product

$$
\mathfrak{P}_{n}=\prod_{p \text { prime: }} p
$$

has been introduced by Kellner and Sondow [13]. Although a priori this could be an infinite product, it is actually a finite product which terminates for $p>(n+1) / 2$, see $[12,13]$.

The relevance of this quantity is due to its link with denominators of Bernoulli polynomials

$$
B_{n}(X)=\sum_{k=0}^{n}\left(\begin{array}{l}
n \\
k
\end{array}\right) B_{k} X^{n-k},
$$

where $B_{k}$ is the $k^{\text {th }}$ Bernoulli number. We also define the polynomials

$$
\widetilde{B}_{n}(X)=B_{n}(X)-B_{n}
$$


which are of interest due to their connection to power sums, namely we have

$$
\sum_{j=1}^{N-1} j^{n-1}=\frac{\widetilde{B}_{n}(N)}{N},
$$

see $[12-14]$. It is shown in $[12,13]$ that the denominator of the polynomial $\widetilde{B}_{n}(X)$ is $\mathfrak{P}_{n}$, thus

$$
\mathfrak{P}_{n} \widetilde{B}_{n}(X) \in \mathbb{Z}[X]
$$

The celebrated von Staudt-Clausen theorem, see [9, Theorem 118], fully describes the denominator of $B_{n}$ for an even $n$ as the product of prime $p$ with $p-1$ dividing $n$ :

$$
\mathfrak{Q}_{n}=\prod_{p: p-1 \mid n} p .
$$

Recall that $B_{1}=1$ and $B_{n}=0$ and thus $\mathfrak{Q}_{n}=1$ for odd $n>1$. One thus sees that the denominator of $B_{n}(X)$ is $\operatorname{lcm}\left[\mathfrak{P}_{n}, \mathfrak{Q}_{n}\right]$.

In this paper, we prove some results about small and large prime factors of $\mathfrak{P}_{n}$. Kellner [12] has also introduced and studied the decomposition

$$
\mathfrak{P}_{n}=\mathfrak{P}_{n}^{-} \cdot \mathfrak{P}_{n}^{+},
$$

where

$$
\mathfrak{P}_{n}^{-}=\prod_{\substack{p<\sqrt{n} \\ s_{p}(n) \geqslant p}} p \quad \text { and } \quad \mathfrak{P}_{n}^{+}=\prod_{\substack{p>\sqrt{n} \\ s_{p}(n) \geqslant p}} p .
$$

Note that the definitions of $\mathfrak{P}_{n}^{ \pm}$, with strict inequalities on $p$ in both, are correct since $s_{p}\left(p^{2}\right)=1$ if $n=p^{2}$ with $p$ a prime. Hence, $p \nmid \mathfrak{P}_{n}$, even if $p=\sqrt{n}$ holds for a prime $p$. Motivated by the link with Bernoulli polynomials Kellner [12] has initiated the study of the arithmetic structure of $\mathfrak{P}_{n}^{-}$and $\mathfrak{P}_{n}^{+}$.

Let, as usual, $\omega(m)$ and $P(m)$ be the number of distinct prime factors of $m$ and the largest prime factor of $m$, respectively.

Conjecture 1.1. (Kellner [12, Conjecture 1].) For $n>192$ we have $P\left(\mathfrak{P}_{n}\right)>\sqrt{n}$.

Conjecture 1.1 is equivalent with the conjecture that for $n>192$ we have $\mathfrak{P}_{n}^{+}>1$ and is established, up to the numerical value of the threshold 192, in a much stronger form in Theorem 1.4. 
Conjecture 1.2. (Kellner [12, Conjecture 2].) There is some absolute constant $\kappa>0$ such that

$$
\omega\left(\mathfrak{P}_{n}^{+}\right)=(\kappa+o(1)) \frac{\sqrt{n}}{\log n}
$$

as $n \rightarrow \infty$.

In this paper we will show that this conjecture is true with $\kappa=$ 2. Moreover, we provide a much sharper estimate (Theorem 1.5) for $\omega\left(\mathfrak{P}_{n}^{+}\right)$than conjectured by Kellner.

Aside from our results on these two conjectures, we improve some of the results on $\mathfrak{P}_{n}$ and $\mathfrak{P}_{n}^{ \pm}$that have been given by Kellner [12] and also obtain several new results.

After introducing some further required notation in Section 1.2, we will state our results in Section 1.3.

1.2. Notation. For a real number $x$ we write $\lfloor x\rfloor$ and $\{x\}$ for its integer and fractional parts, respectively.

For a positive integer $k$ and a positive real number $x$ we write $\log _{k} x$ for the iteratively defined function given by $\log _{1} x=\max \{1, \ln x\}$, where $\ln x$ is a natural $\operatorname{logarithm}$ of $x$ and $\log _{k} x=\max \left\{1, \log _{k-1} x\right\}$ for $k \geqslant 2$. We will also use the functions $\mathbf{e}(x)=\exp (2 \pi i x)$ and $\psi(x)=x-\lfloor x\rfloor-\frac{1}{2}$.

We recall the definitions of $\omega(m)$ and $P(m)$ from Section 1.1 as the number of distinct prime factors of $m$ and the largest prime factor of $m$, respectively. Another standard notation we use is $\pi(x)$ for the counting function of primes $p \leqslant x$.

We also define $\delta$ to be the Erdös-Ford-Tenenbaum constant

$$
\delta=1-(1+\ln \ln 2) / \ln 2=0.08607 \ldots
$$

Throughout the paper, the letters $p$ and $q$ always denote a prime number.

Define

$$
\delta_{c}(x)=e^{-c(\log x)^{3 / 5}\left(\log _{2} x\right)^{-1 / 5}} \quad(x>e, c>0) .
$$

Note that $\delta_{c}(x) \log x \ll \delta_{c_{0}}(x)$ for any $0<c_{0}<c$.

The exponential integral is given by

$$
\mathrm{E}_{1}(x)=\int_{x}^{\infty} \frac{e^{-t}}{t} \mathrm{~d} t \quad(x>0),
$$

see [1, Eq. (5.1.1)].

As usual $A=O(B), A \ll B, B \gg A$ are equivalent to $|A| \leqslant c|B|$ for some absolute constant $c>0$, whereas $A=o(B)$ means that $A / B \rightarrow 0$. 
1.3. Results. Our first result shows that all "small" primes $p$, with at most one exception, divide $\mathfrak{P}_{n}$, where "small" depends on $n$ in a way which is made precise in the following statement.

Theorem 1.3. For any fixed $\varepsilon>0$ there exists $n_{\varepsilon}$ such that for all $n \geqslant$ $n_{\varepsilon}$, all primes $p \leqslant(1 / 2-\varepsilon) \log _{2} n / \log _{3} n$, with at most one exception, divide $\mathfrak{P}_{n}$.

Remark 1. As $2 \nmid \mathfrak{P}_{2^{n}}$, we see that the exceptional prime sometimes exists.

Next we obtain reasonably tight upper and lower bounds on the number of prime divisors and the largest prime divisor of $\mathfrak{P}_{n}$.

Theorem 1.4. We have

$$
P\left(\mathfrak{P}_{n}\right) \geqslant P\left(\mathfrak{P}_{n}^{+}\right) \gg n^{20 / 37} .
$$

This result implies that there exists $n_{0}$ such that Conjecture 1.1 is true with 192 replaced by $n_{0}$.

It is useful to recall that we always have $P\left(\mathfrak{P}_{n}\right)<n / 2+1$ (see $[12$, $13])$, and it is easy to see that for any prime $p$ we have $P\left(\mathfrak{P}_{2 p-1}\right)=p$.

We establish a stronger form of Conjecture 1.2.

Theorem 1.5. There exists $c>0$ such that, for any positive integer $n$ sufficiently large,

$$
\omega\left(\mathfrak{P}_{n}^{+}\right)=n \mathrm{E}_{1}(\log \sqrt{n})+O\left(\sqrt{n} \delta_{c}(\sqrt{n})\right),
$$

where the exponential integral is defined in (1.3) and the function $\delta_{c}$ is given in (1.2).

Successive integration by parts yield that for any positive integer $N \geqslant 1$

$$
\mathrm{E}_{1}(x)=\frac{e^{-x}}{x} \sum_{m=0}^{N-1} \frac{(-1)^{m} m !}{x^{m}}+(-1)^{N} N ! \int_{x}^{\infty} \frac{e^{-t}}{t^{N+1}} \mathrm{~d} t,
$$

from which we immediately deduce the following estimate.

Corollary 1.6. For all positive integers $n, N$, with $n$ sufficiently large,

$$
\omega\left(\mathfrak{P}_{n}^{+}\right)=\sum_{j=1}^{N} \frac{(-1)^{j-1} 2^{j}(j-1) ! \sqrt{n}}{(\log n)^{j}}+O\left(\frac{2^{N+1} N ! \sqrt{n}}{(\log n)^{N+1}}\right),
$$

in particular Conjecture 1.2 holds true with $\kappa=2$.

The following estimate is derived in a similar way. 
Theorem 1.7. There exists a constant $c>0$ such that asymptotically

$$
\log \mathfrak{P}_{n}^{+}=\sqrt{n}+O\left(\sqrt{n} \delta_{c}(\sqrt{n})\right),
$$

where the function $\delta_{c}$ is defined in (1.2).

Finally, we look at how $\mathfrak{P}_{n}$ changes as we move from $n$ to $n+1$. Since $\mathfrak{P}_{n}$ tends to infinity with $n$ by Theorems 1.3 and 1.4, it follows that the inequality $\mathfrak{P}_{n+1}>\mathfrak{P}_{n}$ holds infinitely often. Surprisingly though, the reverse inequality is much more frequent and in fact even in a strict sense, namely we have $\mathfrak{P}_{n}>\mathfrak{P}_{n+1}$ with frequency about $\ln 2=0.6931 \ldots$ However, we also show that the equality $\mathfrak{P}_{n}=\mathfrak{P}_{n+1}$ holds for infinitely many $n$ as well.

Theorem 1.8. For any $x \geqslant 3$ we have:

(i) the divisibility $\mathfrak{P}_{n+1} \mid \mathfrak{P}_{n}$ holds for all except maybe at most $O\left(x\left(\log _{2} x\right)^{-\delta}\left(\log _{3} x\right)^{-1 / 2}\right)$ positive integers $n \leqslant x$;

(ii) the divisibility $\mathfrak{P}_{n+1} \mid \mathfrak{P}_{n}$ and the inequality $\mathfrak{P}_{n}>\mathfrak{P}_{n+1}$ hold simultaneously for at least $(\ln 2+o(1)) x$ positive integers $n \leqslant x$ as $x \rightarrow \infty$;

(iii) the equality $\mathfrak{P}_{q}=\mathfrak{P}_{q+1}$ holds for all except maybe at most $O\left(\pi(x)\left(\log _{2} x\right)^{-c}\right)$ primes $q \leqslant x$, where $c>0$ is an absolute constant.

We remark that Kellner and Sondow [14, Theorem 4] have shown that for odd $n \geqslant 1$ the quotient $\mathfrak{P}_{n} / \mathfrak{P}_{n+1}$ is an odd integer, except that $\mathfrak{P}_{n} / \mathfrak{P}_{n+1}=2$ if $n=2^{k}-1$ for some $k \geqslant 2$. One can also find in $[14]$ several more results about the possible values of the ratios $\mathfrak{P}_{n} / \mathfrak{P}_{n+1}$ for $n$ of special structure.

1.4. Underlying techniques. It is probably interesting to note that in our approach we use a combination of various elementary, Diophantine and analytic techniques.

In particular, for the proof of Theorem 1.3 we employ lower bounds of linear forms in logarithms due to Matveev [16].

For the proof of Theorem 1.4 we use a result about the distribution of fractional parts of reciprocals of primes as well as bounds of exponential sums with reciprocals due to Baker and Harman $[2,3]$.

Finally, we use a recent improvement due to McNew, Pollack and Pomerance [17] of a result of Erdős and Wagstaff [6] on the count of positive integers $n$ divisible by shifted primes (see also [7]), as well as a result of Luca, Pizarro-Madariaga and Pomerance [15] about shifted primes divisible by another shifted prime. 


\section{Proof of Theorem 1.3}

2.1. Sums of digits of integers in different bases. Let $a, b \geqslant 2$ be fixed multiplicatively independent integers. It is shown by Senge and Straus [18], that if $K$ is any fixed number, then there are only finitely many positive integers $n$ such that the sum of digits of $n$ in both bases $a$ and $b$ is at most $K$. This has been made effective by Stewart [19] who, in particular, gives a lower bound

$$
s_{a}(n)+s_{b}(n)>\frac{\log _{2} n}{\log _{3} n+C(a, b)}-1
$$

for all $n>25$, where $C(a, b)$ is some constant depending on $a$ and $b$.

The constant $C(a, b)$ is not made explicit in [19]. Here we do so, as this is important for our purposes, and may also be of independent interest. As in [19], our approach is based on lower bounds for linear forms in logarithms, where we use the bound of Matveev [16]. We only need it for logarithms of rational numbers rather than in its full generality for logarithms of algebraic numbers. We note that for us only the asymptotic dependence of $C(a, b)$ on $a$ and $b$ is important, but we also use this as an opportunity to derive a completely explicit expression for $C(a, b)$.

Let $\rho=r / s$ be a rational number in reduced form $(\operatorname{so},(\operatorname{gcd}(r, s)=1$ and $s \geqslant 1$ ). Then its height is defined as

$$
h(r / s)=\max \{\log |r|, \log s\} .
$$

Let $\alpha_{1}, \ldots, \alpha_{k}$ be rational numbers not zero or \pm 1 . We put $A_{i}=h\left(\alpha_{i}\right)$ for $i=1, \ldots, k$. We let $d_{1}, \ldots, d_{k}$ be nonzero integers and denote $\max \left\{\left|d_{1}\right|, \ldots,\left|d_{k}\right|\right\}$ by $D$. Let

$$
\Lambda=\prod_{i=1}^{k} \alpha_{i}^{d_{i}}-1
$$

The result below follows from [16, Corollary 2.3], and the details have been worked out as [5, Theorem 9.4].

Lemma 2.1. If $\Lambda \neq 0$, then

$$
\log |\Lambda|>-1.4 \cdot 30^{k+3} k^{4.5}(1+\log D) \prod_{i=1}^{k} A_{i} .
$$

We are now ready to present a more explicit version of inequality (2.1). 
Lemma 2.2. Assume that $a$ and $b$ are coprime integers $\geqslant 2$. Let $B \geqslant$ $\max \{a, b\}$. Then the inequality

$$
s_{a}(n)+s_{b}(n)>\frac{\log _{2} n}{\log _{3} n+C(a, b)}
$$

holds with $C(a, b)=\log \left(2 \cdot 10^{12}(\log B)^{2}\right)$ for all $n>\exp \left(10^{15}(\log B)^{4}\right)$.

Proof. We follow [19, pp. 66-69] with the appropriate modifications.

Consider the following $a$-ary and $b$-ary expansions of $n>a+b$ :

$$
\begin{aligned}
& n=a_{1} a^{m_{1}}+a_{2} a^{m_{2}}+\cdots+a_{r} a^{m_{r}}, \quad a_{i} \in\{1, \ldots, a-1\}, \quad 1 \leqslant i \leqslant r, \\
& n=b_{1} b^{\ell_{1}}+b_{2} b^{\ell_{2}}+\cdots+b_{a} b^{\ell_{t}}, \quad b_{i} \in\{1, \ldots, b-1\}, \quad 1 \leqslant i \leqslant t,
\end{aligned}
$$

where

$$
m_{1}>\cdots>m_{r} \geqslant 0 \text { and } \ell_{1}>\ell_{2}>\cdots>\ell_{t} \geqslant 0 \text {. }
$$

We put

$$
\vartheta=c_{0} \log _{2} n,
$$

with $c_{0}$ an explicit constant depending on $B$ to be found later. We now consider the intervals

$$
\Theta_{1}=(0, \vartheta], \quad \Theta_{2}=\left(\vartheta, \vartheta^{2}\right], \quad \ldots, \quad \Theta_{k}=\left(\vartheta^{k-1}, \vartheta^{k}\right],
$$

where $k$ satisfies the inequalities

$$
\vartheta^{k} \leqslant \frac{\log n}{4 \log B}<\vartheta^{k+1}
$$

We assume $k \geqslant 1$ in (2.3). We now show that for an appropriate $c_{0}$ and sufficiently large $n$ each interval $\Theta_{s}$ contains either a term of the form $m_{1}-m_{i}$ for $i=2, \ldots, r$ or a term of the form $\ell_{1}-\ell_{j}$ for $j=2, \ldots, t$. Let us suppose that it is not so. Then there is $s$ with $1 \leqslant s \leqslant k$ such that $\Theta_{s}$ does not contain any $m_{1}-m_{i}$ and any $\ell_{1}-\ell_{j}$. So, let $u, v$ be given by

$$
\begin{aligned}
& m_{1}-m_{u} \leqslant \vartheta^{s-1}, \quad m_{1}-m_{u+1} \geqslant \vartheta^{s} ; \\
& \ell_{1}-\ell_{v} \leqslant \vartheta^{s-1}, \quad \ell_{1}-\ell_{v+1} \geqslant \vartheta^{s} .
\end{aligned}
$$

Note that, since $n>B^{2}$,

$$
m_{1}=\left\lfloor\frac{\log n}{\log a}\right\rfloor \geqslant \frac{\log n}{\log a}-1>\frac{\log n}{4 \log B}>\vartheta^{k} \geqslant \vartheta^{s}
$$

and a similar inequality holds for $\ell_{1}$. Write

$$
n=a^{m_{u}} \alpha_{a}+\zeta_{a} \quad \text { and } \quad n=b^{\ell_{v}} \beta_{b}+\zeta_{b},
$$

with

$$
\alpha_{a}=a_{1} a^{m_{1}-m_{u}}+\cdots+\alpha_{a} \quad \text { and } \quad \beta_{b}=b_{1} b^{\ell_{1}-\ell_{v}}+\cdots+\beta_{b} .
$$


Clearly, $\zeta_{a} \in\left[0, a^{m_{u+1}+1}\right)$ and $\zeta_{b} \in\left[0, b^{\ell_{v+1}+1}\right)$. We then have

$$
\left|\alpha_{a} a^{m_{u}}-\beta_{b} b^{\ell_{v}}\right| \leqslant \max \left\{\zeta_{a}, \zeta_{b}\right\} \leqslant \max \left\{a^{m_{u+1}+1}, b^{\ell_{v+1}+1}\right\} .
$$

We show that the left hand side of (2.6) is nonzero. In order to do so, recall that

SO

$$
m_{1}-m_{u} \leqslant \vartheta^{s-1}<\vartheta^{k}<\frac{\log n}{4 \log B}
$$

$$
\begin{aligned}
m_{u} & =m_{1}-\left(m_{1}-m_{u}\right)=\left\lfloor\frac{\log n}{\log a}\right\rfloor-\frac{\log n}{4 \log B} \\
& \geqslant \frac{\log n}{\log a}-1-\frac{\log n}{4 \log B} \geqslant \frac{3 \log n}{4 \log a}-1>\frac{\log n}{2 \log a},
\end{aligned}
$$

provided $n>B^{4}$. In particular, $a^{m_{u}}>\sqrt{n}$. A similar argument shows that $b^{\ell_{v}}>\sqrt{n}$. Since $a$ and $b$ are coprime, it follows that if the left hand side of (2.6) is zero, then $a^{m_{u}} \alpha_{a}=b^{\ell_{v}} \beta_{b}$, so $a^{m_{u}} \mid \beta_{b}$ and $b^{\ell_{v}} \mid \alpha_{a}$. In particular, both $\alpha_{a}$ and $a^{m_{u}}$ exceed $\sqrt{n}$, so

$$
n=a^{m_{u}} \alpha_{a}+\zeta_{a} \geqslant a^{m_{u}} \alpha_{a}>\sqrt{n} \cdot \sqrt{n}=n,
$$

a contradiction. Assuming that the maximum on the right hand side of $(2.6)$ is $b^{\ell_{v+1}+1}$, we divide both sides of $(2.6)$ by $B b^{\ell_{v}}$, getting

$$
\left|(A / B) a^{m_{u}} b^{-\ell_{v}}-1\right|<\frac{b^{\ell_{v+1}+1}}{b^{\ell_{v}} \beta_{b}} \leqslant \frac{1}{b^{\ell_{v}-\ell_{v+1}-1}} .
$$

A similar inequality holds when the maximum on the right hand side of (2.6) is $a^{m_{u+1}+1}$, namely

$$
\left|(B / A) a^{-m_{u}} b^{\ell_{v}}-1\right|<\frac{1}{a^{m_{1}-m_{u+1}-1}} .
$$

So, we are all set to apply Lemma 2.1 to find a lower bound on the left hand side of (2.7) or (2.8).

We take

$$
l=3, \quad \alpha_{1}=(A / B), \quad \alpha_{2}=a m, \quad \alpha_{3}=b,
$$

and

$$
\left(d_{1}, d_{2}, d_{3}\right)=\varepsilon\left(1, m_{u},-l_{v}\right),
$$

where $\varepsilon \in\{ \pm 1\}$. More precisely, we set

- $\varepsilon=1$ if in (2.6) we have $\max \left\{a^{m_{u+1}+1}, b^{\ell_{v+1}+1}\right\}=b^{\ell_{v+1}+1}$ (and thus (2.7) holds);

- $\varepsilon=-1$ if in (2.6) we have $\max \left\{a^{m_{u+1}+1}, b^{\ell_{v+1}+1}\right\}=a^{m_{u+1}+1}$ (and thus (2.8) holds). 
Since by assumption $a$ and $b$ are multiplicatively independent, these events are clearly mutually exclusive, and so the choice of $\varepsilon$ is welldefined.

Obviously, we have

$$
\max \left\{m_{u}, \ell_{v}\right\}=\max \left\{\left\lfloor\frac{\log n}{\log a}\right\rfloor,\left\lfloor\frac{\log n}{\log b}\right\rfloor\right\} \leqslant \frac{\log n}{\log 2}<2 \log n,
$$

so we can take $D=2 \log n$. Furthermore, $A_{2}=\log a$ and $A_{3}=\log b$. As for $A_{1}$, we have

$$
\begin{aligned}
h\left(\alpha_{1}\right) & \leqslant \max \left\{\log \alpha_{a}, \log \beta_{b}\right\} \leqslant \max \left\{\log \left(a^{m_{1}-m_{u}+1}\right), \log \left(b^{\ell_{1}-\ell_{v}+1}\right)\right\} \\
& \leqslant \max \left\{m_{1}-m_{u}+1, \ell_{1}-\ell_{v}+1\right\} \log B \leqslant\left(\vartheta^{s-1}+1\right) \log B \\
& \leqslant 2 \vartheta^{s-1} \log B
\end{aligned}
$$

so we take $A_{1}=2 \vartheta^{s-1} \log B$. Now Lemma 2.1 combined with inequalities (2.7) or (2.8) tells us that

$$
\begin{aligned}
-1.4 \times 30^{6} \times 3^{4.5}(1 & +\log (2 \log n)) 2 \vartheta^{s-1}(\log a)(\log b)(\log B) \\
& <-\max \left\{\log \left(a^{m_{1}-m_{u+1}-1}\right), \log \left(b^{\ell_{1}-\ell_{v+1}-1}\right)\right\} .
\end{aligned}
$$

Assuming that $n>230$ (so that $2 \log _{2} n>1+\log (2 \log n)$ ), we derive

$$
\begin{aligned}
\max \left\{\left(m_{1}\right.\right. & \left.\left.-m_{u+1}-1\right) \log a,\left(\ell_{1}-\ell_{v}-1\right) \log b\right\} \\
& <6 \cdot 10^{11} \vartheta^{s-1}(\log a)(\log b)(\log B)\left(\log _{2} n\right) .
\end{aligned}
$$

Since by inequality $(2.4)$

$$
\max \left\{m_{1}-m_{u+1}-1, \ell_{1}-\ell_{v+1}-1\right\} \geqslant \vartheta^{s}-1>\frac{\vartheta^{s}}{2}
$$

for $s \geqslant 1$, we see that

$$
\frac{\vartheta^{s}}{2}<6 \cdot 10^{11} \vartheta^{s-1}(\log B)^{2} \log _{2} n,
$$

giving

$$
\vartheta<12 \cdot 10^{11}(\log B)^{2} \log _{2} n,
$$

which is false if we choose $c_{0}=2 \cdot 10^{12}(\log B)^{2}$. It remains to establish the starting value for $n$ such that $k \geqslant 1$. That is,

$$
\vartheta<\frac{\log n}{4 \log B} .
$$

This is equivalent to

$$
\frac{\log n}{\log _{2} n}>4 c_{0}(\log B)^{3}
$$


The right hand side above is $8 \cdot 10^{12}(\log B)^{3}$. The inequality

$$
\frac{x}{\log x}>A
$$

is fulfilled when $x>2 A \log A$, provided that $A>e$. We now take $A=10^{13}(\log B)^{3}$ and we see that $(2.9)$ holds, provided that

$$
\log n>2 \cdot 10^{13}(\log B)^{3}\left(13 \log 10+3 \log _{2} B\right) .
$$

Since $\log _{2} B<\log B, B \geqslant 3$, and $13 \log 10+3<33$, it follows that the desired inequality holds for $\log n>10^{15}(\log B)^{4}$. Thus indeed, each interval $\Theta_{s}$ for $s=1, \ldots, k$ contains one of $m_{1}-m_{i}$ or $\ell_{1}-\ell_{j}$ for $i=1, \ldots, r$, or $j=1, \ldots, t$. Hence, $r-1+s-1 \geqslant k$. Therefore,

$$
\begin{aligned}
s_{a}(n)+s_{b}(n) & \geqslant r+s \geqslant k+2=(k+1)+1 \\
& >\frac{\log ((\log n) /(4 \log B))}{\log \vartheta}+1 \\
& =\frac{\log _{2} n+(\log \vartheta-\log (4 \log B))}{\log _{3} n+\log c_{0}},
\end{aligned}
$$

on recalling that $\vartheta=c_{0} \log \log n$. Clearly, $\vartheta>4 \log B$. Thus the inequality

$$
s_{a}(n)+s_{b}(n)>\frac{\log _{2} n}{\log _{3} n+C}
$$

holds with $C=\log \left(2 \cdot 10^{12}(\log B)^{2}\right)$, which concludes the proof.

2.2. The proof of Theorem 1.3. Let $\varepsilon>0$ be arbitrary. We consider the primes $p \leqslant B$ with $B=\log _{2} n$. Then, as $n \rightarrow \infty$, we have

$$
C=\log \left(2 \cdot 10^{12}(\log B)^{2}\right)=2 \log _{4} n+O(1) .
$$

Further, we need to check that $n>\exp \left(10^{15}(\log B)^{4}\right)$. This is equivalent to $\log n>10^{15}\left(\log _{3} n\right)^{4}$, which holds for all $n>\exp \left(10^{18}\right)$. Thus, assuming $n$ is this large, for any two distinct primes $p, q \leqslant B$, it follows by inequality (2.1) that, as $n \rightarrow \infty$, the inequality

$$
s_{p}(n)+s_{q}(n)>(1+o(1)) \frac{\log _{2} n}{\log _{3} n}
$$

holds uniformly in primes $p, q \leqslant B$. If in particular we take $P=$ $(1 / 2-\varepsilon) \log _{2} n / \log _{3} n$, it follows that for every $n>n_{\varepsilon}$, there is at most one prime $q \leqslant \log _{2} n$ such that

$$
s_{q}(n)<P .
$$

For all other primes $p \leqslant \log _{2} n$, we then have $s_{p}(n) \geqslant P$. So, if in addition we also have $p<P$, then $s_{p}(n) \geqslant P>p$, so $p \mid \mathfrak{P}_{n}$. Thus, 
indeed, for all $\varepsilon>0$, there exists $n_{\varepsilon}$ such that if $n>n_{\varepsilon}$, then all primes $p<(1 / 2-\varepsilon) \log _{2} n / \log _{3} n$ divide $\mathfrak{P}_{n}$ with at most one exception.

\section{The PRoOF of TheOREM 1.4}

3.1. Fractional parts of reciprocals of primes. We will use the following result from [2, Proposition 2]:

Lemma 3.1. For all $v$ and $w$ that satisfy

$$
v^{37 / 20} \leqslant w \leqslant v^{2}
$$

and are sufficiently large, we have

$$
\#\left\{p: 2 v<p<3 v,\left\{\frac{w}{p}\right\} \geqslant 1-\frac{w}{16 v^{2}}\right\} \gg \frac{w}{v \log w} .
$$

3.2. Concluding the proof. We now derive one of our main technical results.

Lemma 3.2. For all $n$ and $v$ that satisfy

$$
v^{37 / 20} \leqslant n \leqslant v^{2}
$$

and are sufficiently large, we have

$$
\#\left\{p: 2 v<p<3 v, p \mid \mathfrak{P}_{n}^{+}\right\} \gg \frac{n}{v \log n} .
$$

Proof. Let $p$ be a prime counted in Lemma 3.1 taken with $w=n$, that is,

$$
1>\left\{\frac{n}{p}\right\} \geqslant 1-\frac{n}{16 v^{2}} .
$$

Clearly, $p^{2}>4 v^{2}>n$. Thus, writing $n$ in base $p$, we have $n=a p+b$, where

and

$$
a=\lfloor n / p\rfloor \geqslant n / p-1
$$

$$
b=p\left\{\frac{n}{p}\right\} \geqslant p-\frac{n p}{16 v^{2}} .
$$

Thus,

$$
\begin{aligned}
s_{p}(n) & =a+b \geqslant p+\frac{n}{p}-\frac{n p}{16 v^{2}}-1=p+\frac{n}{p}\left(1-\frac{p^{2}}{16 v^{2}}\right)-1 \\
& >p+\frac{n}{p}\left(1-\frac{9}{16}\right)-1=p+\frac{7 n}{16 p}-1>p,
\end{aligned}
$$

assuming $p$ (and hence, $n$ ) is sufficiently large. 
Now we are home and dry.

The proof of Theorem 1.4. Apply Lemma 3.2 with $v=n^{20 / 37}$.

\section{Proof of Theorems 1.5 And 1.7}

4.1. Preliminary comments. The proofs of both Theorems 1.5 and 1.7 may be unified in only one proof by making use of the number $\kappa \in\{0,1\}$. Indeed, our method also works if the number $\kappa$ is any real non-negative number. However, this generalization does not seem to bring added value and is left to the interested reader.

4.2. Tools. We start with the following simple result.

Lemma 4.1. Let $\kappa \in\{0,1\}$. For any real numbers $\varepsilon \in(0,1), x \geqslant 2$ and $1<x^{\varepsilon} \leqslant y \leqslant z<x$

$$
\sum_{z<p \leqslant x}\left(\left\lfloor\frac{x+y}{p}\right\rfloor-\left\lfloor\frac{x}{p}\right\rfloor\right)(\log p)^{\kappa}<2 \varepsilon^{-1}(y+1)(\log x)^{\kappa} .
$$

Proof. As $\log p \leqslant \log x$ for $p \leqslant x$, it suffices to show that the inequality holds if $\kappa=0$. We write

$$
\sum_{z<p \leqslant x}\left(\left\lfloor\frac{x+y}{p}\right\rfloor-\left\lfloor\frac{x}{p}\right\rfloor\right)=\sum_{z<p \leqslant x} \sum_{x / p<m \leqslant(x+y) / p} 1 .
$$

Collecting together products $k=m p \in(x, x+y]$ and changing the order of summation we obtain

$$
\sum_{z<p \leqslant x}\left(\left\lfloor\frac{x+y}{p}\right\rfloor-\left\lfloor\frac{x}{p}\right\rfloor\right)=\sum_{x<k \leqslant x+y} \sum_{\substack{p \mid k \\ z<p \leqslant x}} 1 \leqslant \sum_{x<k \leqslant x+y} \frac{\log k}{\log z} .
$$

Since for $k \in(x, x+y]$ we have

$$
\frac{\log k}{\log z} \leqslant \frac{\log (x+y)}{\log z} \leqslant \frac{\log (2 x)}{\log y} \leqslant \frac{2}{\varepsilon},
$$

the result now follows.

Corollary 4.2. Let $\kappa \in\{0,1\}$ and $\alpha \in\left(n^{-7 / 16}, 1\right)$. Then

$$
\sum_{\sqrt{n} / \alpha<p \leqslant n}\left(\left\lfloor\frac{n}{p}+\frac{n-p}{p(p-1)}\right\rfloor-\left\lfloor\frac{n}{p}\right\rfloor\right)(\log p)^{\kappa} \ll \alpha \sqrt{n}(\log n)^{\kappa} .
$$


Proof. If $p>\sqrt{n} / \alpha$, then

$$
\alpha \sqrt{n}-\frac{n-p}{p-1}>\alpha \sqrt{n}-\frac{n-\sqrt{n} / \alpha}{\sqrt{n} / \alpha-1}=\frac{\sqrt{n}(1+\alpha)(1-\alpha)}{\sqrt{n}-\alpha}>0,
$$

where we used that $(n-p) /(p-1)=(n-1) /(p-1)-1$ is a decreasing function for $p \geqslant 2$. Consequently,

$$
\begin{aligned}
\sum_{\sqrt{n} / \alpha<p \leqslant n} & \left(\left\lfloor\frac{n}{p}+\frac{n-p}{p(p-1)}\right\rfloor-\left\lfloor\frac{n}{p}\right\rfloor\right)(\log p)^{\kappa} \\
& \leqslant \sum_{\sqrt{n} / \alpha<p \leqslant n}\left(\left\lfloor\frac{n+\alpha \sqrt{n}}{p}\right\rfloor-\left\lfloor\frac{n}{p}\right\rfloor\right)(\log p)^{\kappa},
\end{aligned}
$$

and the proof is achieved on invoking Lemma 4.1 with $x=n, y=\alpha \sqrt{n}$, $z=\sqrt{n} / \alpha$ and $\varepsilon=1 / 16$.

Lemma 4.3. Let $\kappa \in\{0,1\}$ and let $M$ be a positive integer. For any real valued function $g: \mathbb{Z} \rightarrow \mathbb{R}$ and any positive integer $H$ we have

$$
\begin{aligned}
& \left|\sum_{M<p \leqslant 2 M}(\log p)^{\kappa} \psi\left(\frac{n}{p}+g(p)\right)\right| \\
& \quad \ll \frac{M}{H(\log M)^{1-\kappa}}+\sum_{h \leqslant H} \frac{1}{h}\left|\sum_{M<p \leqslant 2 M}(\log p)^{\kappa} \mathbf{e}\left(\frac{n h}{p}\right) \mathbf{e}(h g(p))\right|
\end{aligned}
$$

Proof. For any $0<|t|<1$ we put $\Phi(t)=\pi t(1-|t|) \cot (\pi t)+|t|$. Note that $0<\Phi(t)<1$ for $0<|t|<1$.

We follow the proof of [4, Corollary 6.2]. It follows from the result of Vaaler [20], which we use in the form given by [4, Theorem 6.1], that for any real number $x \geqslant 1$ and any positive integer $H$,

$$
\psi(x)=-\sum_{0<|h| \leqslant H} \Phi\left(\frac{h}{H+1}\right) \frac{\mathbf{e}(h x)}{2 \pi i h}+\mathcal{R}_{H}(x),
$$

where the error term $\mathcal{R}_{H}(x)$ satisfies

$$
\left|\mathcal{R}_{H}(x)\right| \leqslant \frac{1}{2 H+2} \sum_{|h| \leqslant H}\left(1-\frac{|h|}{H+1}\right) \mathbf{e}(h x) .
$$

The right-hand side of (4.2) does not look like, but is in fact a non-zero real number since it can be shown, cf. [4, Exercise 3, p. 350], that

$$
\sum_{|h| \leqslant H}\left(1-\frac{|h|}{H+1}\right) \mathbf{e}(h x)=\frac{1}{H+1}\left|\sum_{h=0}^{H} \mathbf{e}(h x)\right|^{2} .
$$


Using (4.1) with $x=n / p+g(p)$, multiplying by $(\log p)^{\kappa}$ and summing over all the primes $p$ in $(M, 2 M]$ yields the estimate

$$
\sum_{M<p \leqslant 2 M}(\log p)^{\kappa} \psi\left(\frac{n}{p}+g(p)\right)=\Sigma_{1}+\Sigma_{2},
$$

where

$$
\begin{aligned}
& \Sigma_{1}=-\sum_{0<|h| \leqslant H} \Phi\left(\frac{h}{H+1}\right) \frac{1}{2 \pi i h} \sum_{M<p \leqslant 2 M}(\log p)^{\kappa} \mathbf{e}\left(\frac{n h}{p}+h g(p)\right), \\
& \Sigma_{2}=\sum_{M<p \leqslant 2 M}(\log p)^{\kappa} \mathcal{R}_{H}\left(\frac{n}{p}+g(p)\right) .
\end{aligned}
$$

Now

$$
\begin{aligned}
\left|\Sigma_{1}\right| & \leqslant \sum_{0<|h| \leqslant H} \frac{1}{2 \pi|h|}\left|\sum_{M<p \leqslant 2 M}(\log p)^{\kappa} e\left(\frac{n h}{p}+h g(p)\right)\right| \\
& =\frac{1}{\pi} \sum_{h \leqslant H} \frac{1}{h}\left|\sum_{M<p \leqslant 2 M}(\log p)^{\kappa} \mathbf{e}\left(\frac{n h}{p}\right) e(h g(p))\right|,
\end{aligned}
$$

and by (4.2) we have

$$
\begin{aligned}
\left|\Sigma_{2}\right| \leqslant & \sum_{M<p \leqslant 2 M}(\log p)^{\kappa}\left|\mathcal{R}_{H}\left(\frac{n}{p}+g(p)\right)\right| \\
\leqslant & \frac{1}{2 H+2} \sum_{|h| \leqslant H}\left(1-\frac{|h|}{H+1}\right) \sum_{M<p \leqslant 2 M}(\log p)^{\kappa} e\left(\frac{n h}{p}+h g(p)\right) \\
= & \frac{1}{2 H+2} \sum_{M<p \leqslant 2 M}(\log p)^{\kappa}+\frac{1}{H+1} \sum_{h \leqslant H}\left(1-\frac{h}{H+1}\right) \\
& \times \frac{1}{2 H+2} \sum_{M<p \leqslant 2 M}\left(\sum_{M<p \leqslant 2 M}(\log p)^{\kappa} \mathbf{e}\left(\frac{n h}{p}+h g(p)\right)\right) \\
& +\sum_{h \leqslant H} \frac{1}{h} \mid \sum_{M<p \leqslant 2 M}(\log p)^{\kappa}
\end{aligned}
$$

where in the third line the cases $h=0$ and $|h|>0$ are separated, concluding the proof. 
Recall that the von Mangoldt function is defined by

$$
\Lambda(m)= \begin{cases}\log p & \text { if } m \text { is a power of a prime } p \\ 0 & \text { otherwise. }\end{cases}
$$

Lemma 4.4. Let $\kappa \in\{0,1\}$ and let $n \geqslant 1$ and $M \geqslant 2$ be positive integers.

For a prime $p$, define

$$
g(p)=0 \quad \text { or } \quad g(p)=\frac{n-p}{p(p-1)} .
$$

Then for any positive integer $H$ we have

$$
\begin{gathered}
\left|\sum_{M<p \leqslant 2 M}(\log p)^{\kappa} \psi\left(\frac{n}{p}+g(p)\right)\right| \\
\ll \frac{1}{(\log M)^{1-\kappa}} \sum_{h \leqslant H} \frac{1}{h}\left(1+\frac{n h}{M^{2}}\right) \max _{M<N \leqslant 2 M}\left|\sum_{M<m \leqslant N} \Lambda(m) \mathbf{e}\left(\frac{h n}{m}\right)\right| \\
\quad+\frac{M}{H(\log M)^{1-\kappa}}+\frac{n H}{M^{3 / 2}(\log M)^{1-\kappa}}+\frac{\sqrt{M} \log H}{(\log M)^{1-\kappa}} .
\end{gathered}
$$

Proof. From Lemma 4.3,

$$
\begin{aligned}
& \left|\sum_{M<p \leqslant 2 M}(\log p)^{\kappa} \psi\left(\frac{n}{p}+g(p)\right)\right| \\
& \quad \ll \frac{1}{H} \sum_{M<p \leqslant 2 M}(\log p)^{\kappa}+\sum_{h \leqslant H} \frac{1}{h}\left|\sum_{M<p \leqslant 2 M}(\log p)^{\kappa} \mathbf{e}\left(\frac{n h}{p}\right) \mathbf{e}(h g(p))\right| \\
& \ll \frac{M}{H(\log M)^{1-\kappa}}+\sum_{h \leqslant H} \frac{1}{h}\left|\sum_{M<p \leqslant 2 M}(\log p)^{\kappa} \mathbf{e}\left(\frac{n h}{p}\right) \mathbf{e}(h g(p))\right| .
\end{aligned}
$$

Now since

$$
|h g(p)| \leqslant \frac{n h}{p(p-1)} \leqslant \frac{2 n h}{M^{2}} \quad(M<p \leqslant 2 M),
$$

we get by Abel summation

$$
\begin{aligned}
\left|\sum_{M<p \leqslant 2 M}(\log p)^{\kappa} \mathbf{e}\left(\frac{n h}{p}\right) \mathbf{e}(h g(p))\right| \\
\quad \ll\left(1+\frac{n h}{M^{2}}\right) \max _{M<N \leqslant 2 M}\left|\sum_{M<p \leqslant N}(\log p)^{\kappa} \mathbf{e}\left(\frac{n h}{p}\right)\right|,
\end{aligned}
$$


and by Abel summation again

$$
\begin{aligned}
\left|\sum_{M<p \leqslant N}(\log p)^{\kappa} \mathbf{e}\left(\frac{n h}{p}\right)\right| \\
\quad \leqslant \frac{2}{(\log M)^{1-\kappa}} \max _{M<L \leqslant N}\left|\sum_{M \leqslant p \leqslant L}(\log p) \mathbf{e}\left(\frac{n h}{p}\right)\right| \\
\quad \ll \frac{1}{(\log M)^{1-\kappa}}\left(\max _{M<L \leqslant N}\left|\sum_{M \leqslant m \leqslant L} \Lambda(m) \mathbf{e}\left(\frac{n h}{m}\right)\right|+\sqrt{N}\right) .
\end{aligned}
$$

The asserted estimate follows on putting everything together.

We also recall that using [8, Theorem 9] with $k=2$ we obtain

Lemma 4.5. If $M \leqslant x^{3 / 5} / 5$, then, for any $N \in(M, 2 M]$

$$
\left|\sum_{M<m \leqslant N} \Lambda(m) \mathbf{e}\left(\frac{x}{m}\right)\right|<17\left(x^{2} M^{19}\right)^{1 / 24}(\log 16 M)^{11 / 4} .
$$

Lemma 4.6. Let $\kappa \in\{0,1\}$. There exists some absolute constant $c>0$ such that, for any large real number $t>1$ we have

$$
\sum_{p>t} \frac{(\log p)^{\kappa}}{p(p-1)}=F_{\kappa}(t)+O\left(t^{-1} \delta_{c}(t)\right)
$$

where $F_{0}(t)=\mathrm{E}_{1}(\log t)$ and $F_{1}(t)=t^{-1}$.

Proof. We recall the Prime Number Theorem in the form

$$
\pi(u)=\operatorname{li}(u)+O\left(u \delta_{c_{0}}(u)\right)
$$

where $\operatorname{li}(u)$ is the logarithmic integral

$$
\operatorname{li}(u)=\int_{2}^{u} \frac{\mathrm{d} t}{\log t} \quad(u>0)
$$

the function $\delta_{c_{0}}$ is defined in (1.2) and $c_{0}>0$ is an absolute constant (see [10, Theorem 12.2 and Eq. (12.27)] or [11, Corollary 8.30], for instance). By partial summation and the Prime Number Theorem in 
the above form, we derive

$$
\begin{aligned}
\sum_{p>t} \frac{(\log p)^{\kappa}}{p(p-1)}=\sum_{p>t} \frac{(\log p)^{\kappa}}{p^{2}}+\sum_{p>t} \frac{(\log p)^{\kappa}}{p^{2}(p-1)} & \\
=- & \frac{\pi(t)(\log t)^{\kappa}}{t^{2}}+\int_{t}^{\infty} \frac{\pi(u)\left(2(\log u)^{\kappa}-\kappa(\log u)^{\kappa-1}\right)}{u^{3}} \mathrm{~d} u \\
& \quad+O\left(\frac{1}{t^{2}(\log t)^{1-\kappa}}\right) \\
=- & \frac{(\log t)^{\kappa}}{t^{2}}\left(\operatorname{li}(t)+O\left(t \delta_{c_{0}}(t)\right)\right) \\
& \quad+\int_{t}^{\infty} \frac{2(\log u)^{\kappa}-\kappa(\log u)^{\kappa-1}}{u^{3}}\left(\operatorname{li}(u)+O\left(u \delta_{c_{0}}(u)\right)\right) \mathrm{d} u \\
=G(t) & +O\left(\frac{(\log t)^{\kappa} \delta_{c_{0}}(t)}{t}\right),
\end{aligned}
$$

where

$$
G(t)=-\frac{\operatorname{li}(t)(\log t)^{\kappa}}{t^{2}}+\int_{t}^{\infty} \frac{\operatorname{li}(u)\left(2(\log u)^{\kappa}-\kappa(\log u)^{\kappa-1}\right)}{u^{3}} \mathrm{~d} u .
$$

Now, integrating by parts we derive

$$
G(t)=\int_{t}^{\infty} \frac{\mathrm{d} u}{u^{2}(\log u)^{1-\kappa}}=F_{\kappa}(t) .
$$

The result follows with any constant $c \in\left(0, c_{0}\right)$.

4.3. First step. Let $\kappa \in\{0,1\}$ and define

$$
\Sigma_{\kappa}=\sum_{\sqrt{n}<p \leqslant n}\left(\left\lfloor\frac{n-1}{p-1}\right\rfloor-\left\lfloor\frac{n}{p}\right\rfloor\right)(\log p)^{\kappa} .
$$

Notice that, if $\sqrt{n}<p \leqslant n$, then

$$
0 \leqslant \frac{n-p}{p-1}<\sqrt{n}
$$

and hence,

$$
\frac{n-1}{p-1}-\frac{n}{p}=\frac{n-p}{p(p-1)}<\frac{\sqrt{n}}{p}<1,
$$

so that

$$
\omega\left(\mathfrak{p}_{n}^{+}\right)=\Sigma_{0} \quad \text { and } \quad \log \left(\mathfrak{p}_{n}^{+}\right)=\Sigma_{1} .
$$

We split the sum into two subsums as

$$
\Sigma_{\kappa}=S_{1}+S_{2}
$$


where

$$
\begin{aligned}
& S_{1}=\sum_{\sqrt{n}<p \leqslant \sqrt{n}\left(\delta_{c}(\sqrt{n})\right)^{-1}}\left(\left\lfloor\frac{n-1}{p-1}\right\rfloor-\left\lfloor\frac{n}{p}\right\rfloor\right)(\log p)^{\kappa}, \\
& S_{2}=\sum_{\sqrt{n}\left(\delta_{c}(\sqrt{n})\right)^{-1}<p \leqslant n}\left(\left\lfloor\frac{n-1}{p-1}\right\rfloor-\left\lfloor\frac{n}{p}\right\rfloor\right)(\log p)^{\kappa},
\end{aligned}
$$

and $c>0$ is the constant given in Lemma 4.6.

4.4. The sum $S_{2}$. We use Corollary 4.2 with $\alpha=\delta_{c}(\sqrt{n})$ obtaining immediately

$$
S_{2} \ll \sqrt{n} \delta_{c}(\sqrt{n})(\log n)^{\kappa} \ll \sqrt{n} \delta_{c_{1}}(\sqrt{n})
$$

for some $c_{1} \in(0, c]$.

\subsection{The sum $S_{1}$. Using}

$$
\frac{n-1}{p-1}=\frac{n}{p}+\frac{n-p}{p(p-1)},
$$

and recalling the definition of the function $\psi(x)$, we write

$$
S_{1}=S_{11}-S_{12}
$$

where

$$
\begin{aligned}
& S_{11}=\sum_{\sqrt{n}<p \leqslant \sqrt{n}\left(\delta_{c}(\sqrt{n})\right)^{-1}} \frac{(\log p)^{\kappa}(n-p)}{p(p-1)}, \\
& S_{12}=\sum_{\sqrt{n}<p \leqslant \sqrt{n}\left(\delta_{c}(\sqrt{n})\right)^{-1}}(\log p)^{\kappa}\left(\psi\left(\frac{n}{p}+\frac{n-p}{p(p-1)}\right)-\psi\left(\frac{n}{p}\right)\right) .
\end{aligned}
$$

The main term. For any integer $n \geqslant 1$, we derive from Lemma 4.6 that

$$
\sum_{\sqrt{n}<p \leqslant \sqrt{n}\left(\delta_{c}(\sqrt{n})\right)^{-1}} \frac{(\log p)^{\kappa}}{p(p-1)}=F_{\kappa}(\sqrt{n})-F_{\kappa}\left(\frac{\sqrt{n}}{\delta_{c}(\sqrt{n})}\right)+O\left(\frac{\delta_{c}(\sqrt{n})}{\sqrt{n}}\right) .
$$

From [1, Eq. (5.1.19)], we have the inequalities

$$
\frac{e^{-x}}{x+1}<\mathrm{E}_{1}(x)<\frac{e^{-x}}{x} \quad(x>0)
$$

which imply that

$$
F_{0}\left(\frac{\sqrt{n}}{\delta_{c}(\sqrt{n})}\right) \ll \frac{\delta_{c}(\sqrt{n})}{\sqrt{n} \log \left(n / \delta_{c}(\sqrt{n})^{2}\right)} \ll \frac{\delta_{c}(\sqrt{n})}{\sqrt{n}},
$$


and also

$$
F_{0}\left(\frac{\sqrt{n}}{\delta_{c}(\sqrt{n})}\right) \ll \frac{\delta_{c}(\sqrt{n})}{\sqrt{n}},
$$

so that

$$
\sum_{\sqrt{n}<p \leqslant \sqrt{n}\left(\delta_{c}(\sqrt{n})\right)^{-1}} \frac{(\log p)^{\kappa}}{p(p-1)}=F_{\kappa}(\sqrt{n})+O\left(\frac{\delta_{c}(\sqrt{n})}{\sqrt{n}}\right) .
$$

Therefore,

$$
\begin{aligned}
& S_{11}=n \sum_{\sqrt{n}<p \leqslant \sqrt{n}\left(\delta_{c}(\sqrt{n})\right)^{-1}} \frac{(\log p)^{\kappa}}{p(p-1)}-\sum_{\sqrt{n}<p \leqslant \sqrt{n}\left(\delta_{c}(\sqrt{n})\right)^{-1}} \frac{(\log p)^{\kappa}}{p-1} \\
& =n \sum_{\sqrt{n}<p \leqslant \sqrt{n}\left(\delta_{c}(\sqrt{n})\right)^{-1}} \frac{(\log p)^{\kappa}}{p(p-1)}+O(\log n) \\
& =n F_{\kappa}(\sqrt{n})+O\left(\sqrt{n} \delta_{c}(\sqrt{n})\right) \text {. }
\end{aligned}
$$

The error term. It remains to prove that, for $n$ sufficiently large,

$$
\left|S_{12}\right| \ll \sqrt{n} \delta_{c}(\sqrt{n}) .
$$

This estimate follows from the next result, which actually gives a power saving.

Lemma 4.7. We have $S_{12} \leqslant n^{49 / 100+o(1)}$ as $n \rightarrow \infty$.

Proof. Split the interval $\left(\sqrt{n}, \sqrt{n}\left(\delta_{c}(\sqrt{n})\right)^{-1}\right]$ into $O(\log n)$ dyadic subintervals of the form $(M, 2 M]$, so that

$$
\begin{array}{|}
\left|\sum_{\sqrt{n}<p \leqslant \sqrt{n}\left(\delta_{c}(\sqrt{n})\right)^{-1}}(\log p)^{\kappa} \psi\left(\frac{n}{p}+g(p)\right)\right| \\
\ll \max _{\sqrt{n}<M \leqslant \sqrt{n}\left(\delta_{c}(\sqrt{n})\right)^{-1}}\left|\sum_{M<p \leqslant 2 M}(\log p)^{\kappa} \psi\left(\frac{n}{p}+g(p)\right)\right| \log n,
\end{array}
$$

where, as in Lemma 4.4,

$$
g(p)=0 \quad \text { or } \quad g(p)=\frac{n-p}{p(p-1)} .
$$


From Lemmas 4.4 and 4.5

$$
\begin{gathered}
\left|\sum_{M<p \leqslant 2 M}(\log p)^{\kappa} \psi\left(\frac{n}{p}+g(p)\right)\right| \\
\ll(\log M)^{7 / 4+\kappa} \sum_{h \leqslant H} \frac{1}{h}\left(1+\frac{n h}{M^{2}}\right)\left(h^{2} n^{2} M^{19}\right)^{1 / 24} \\
+\frac{M}{H(\log M)^{1-\kappa}}+\frac{n H}{M^{3 / 2}(\log M)^{1-\kappa}}+\frac{\sqrt{M} \log H}{(\log M)^{1-\kappa}} \\
\ll\left(\left(n^{26} H^{26} M^{-29}\right)^{1 / 24}+\left(n^{2} H^{2} M^{19}\right)^{1 / 24}\right)(\log M)^{11 / 4} \\
+\frac{M}{H(\log M)^{1-\kappa}}+\frac{n H}{M^{3 / 2}}+\sqrt{M} \log H .
\end{gathered}
$$

Choose

$$
H=\left\lfloor M^{53 / 50} n^{-13 / 25}\right\rfloor
$$

to balance the first and the third terms in the above bound up to logarithmic factors, which we all replace by $n^{o(1)}$. We also note that, since $M \geqslant \sqrt{n}$, we have $H \geqslant 1$ provided that $n$ is large enough. Hence,

$$
\begin{aligned}
& \left|\sum_{M<p \leqslant 2 M}(\log p)^{\kappa} \psi\left(\frac{n}{p}+g(p)\right)\right| \\
& \quad \ll\left(\left(n^{26} M^{-3}\right)^{1 / 50}+\left(n M^{22}\right)^{1 / 25}+\left(n^{12} M^{-11}\right)^{1 / 25}+M^{1 / 2}\right) n^{o(1)} .
\end{aligned}
$$

Using $M=n^{1 / 2+o(1)}$ we obtain

$$
\left|S_{12}\right| \ll\left(n^{49 / 100}+n^{13 / 50}+n^{1 / 4}\right) n^{o(1)} \ll n^{49 / 100+o(1)},
$$

as $n \rightarrow \infty$, concluding the proof.

4.6. Completion of the proof of Theorems $\mathbf{1 . 5}$ and 1.7. Follows at once from (4.3), (4.4), (4.5) and (4.6).

\section{The Proof of Theorem 1.8}

5.1. Preliminary considerations. Let us fix some prime $p$ and write $n$ in base $p$ as

$$
n=\sum_{i=0}^{k} a_{i} p^{k-i}
$$

with $p$-ary digits

$$
a_{i} \in\{0, \ldots, p-1\}, \quad i=0, \ldots, k, \quad \text { and } \quad a_{0} \neq 0 .
$$

In the above, $k=\lfloor\log n / \log p\rfloor+1$. 
We distinguish the following two cases:

Case 1: $a_{k} \neq p-1$.

In this case,

$$
n+1=a_{0} p^{k}+\cdots+\left(a_{k}+1\right)
$$

is the base $p$-representation of $n+1$. Hence, $s_{p}(n+1)=s_{p}(n)+1$. In particular, the following events

$$
p \mid \mathfrak{P}_{n+1} \quad \text { and } \quad p \nmid \mathfrak{P}_{n}
$$

and thus,

$$
s_{p}(n+1) \geqslant p>s_{n}(p),
$$

are simultaneously possible only when

$$
s_{p}(n)=p-1 \text {. }
$$

Case 2: $a_{k}=p-1$.

Let $i \in[0, k]$ be such that $a_{k}=a_{k-1}=\cdots=a_{k-i}=p-1$, but $a_{k-i-1}<p-1$. Then

$$
n+1=a_{0} p^{k}+\cdots+\left(a_{k-i-1}+1\right) p^{i}
$$

and we obtain

$$
s_{p}(n+1)=a_{0}+\cdots+a_{k-i-1}+1 \leqslant a_{0}+\cdots+a_{k-i-1}+a_{k} \leqslant s_{p}(n) .
$$

Hence, for each $p$ with $a_{k}=p-1$, if $p \mid \mathfrak{P}_{n+1}$, then we also have $p \mid \mathfrak{P}_{n}$. However, the opposite of (5.1), that is

$$
p \nmid \mathfrak{P}_{n+1} \quad \text { and } \quad p \mid \mathfrak{P}_{n}
$$

is also possible in this case. Actually this case plays an important role in our argument in Section 5.2 below.

5.2. Proof of (i): divisibility. As we have seen, primes $p$ which belong to Case 2 of Section 5.1 do not appear in the denominator of the ratio $\mathfrak{P}_{n} / \mathfrak{P}_{n+1}$. We now show that for almost all $n \leqslant x$ (with an explicit bound on the size of the exceptional set) the primes which belong to Case 1 of Section 5.1 do not appear in the denominator of this ratio either, and thus we have the desired divisibility.

More precisely, using the characterization of (5.1) given by (5.2), we we conclude that $\mathfrak{P}_{n+1} \mid \mathfrak{P}_{n}$ holds for all positive integers $n$ that have the property that there is no prime $p$ with $s_{p}(n)=p-1$. It remains to prove that the complementary set has asymptotic density zero.

Thus, we define

$$
\mathcal{A}(x)=\left\{n \leqslant x: s_{p}(n)=p-1 \text { for some prime } p\right\} .
$$


First of all note that since $s_{p}(n)=p-1$, it follows that $(p-1) \mid n$. McNew, Pollack and Pomerance [17], improving on the previous result of Erdös and Wagstaff [6], have shown that uniformly in $3 \leqslant y \leqslant x$,

$$
\#\{n \leqslant x:(p-1) \mid n \text { for some } p \geqslant y\} \ll \frac{x}{(\log y)^{\delta}\left(\log _{2} y\right)^{1 / 2}},
$$

where $\delta$ is the Erdős-Ford-Tenenbaum constant defined in (1.1). We also recall that Ford [7] has recently established more precise results, which however cannot be used to improve our bounds.

We take $y=\sqrt{\log x}$ in (5.4), getting that the number of $n \in \mathcal{A}(x)$ such that $s_{p}(n)=p-1$ for some $p \geqslant y$ is

$$
\frac{x}{(\log y)^{\delta} \sqrt{\log _{2} y}} \ll \frac{x}{\left(\log _{2} x\right)^{\delta} \sqrt{\log _{3} x}} .
$$

Assume now that $p<y$. We remark that for each $p$ and

$$
k=\left\lfloor\frac{\log n}{\log p}\right\rfloor+1
$$

the condition $s_{p}(n)=p-1$ leads to the equation

$$
a_{0}+\cdots+a_{k}=p-1
$$

on the $p$-ary digits $a_{0}, \ldots, a_{k}$ of $n$ (we possibly append some leading zeros to make all $p$-ary expansions of the same length). Thus, for each $p$ there are at most

$$
\left(\begin{array}{c}
k+p \\
p
\end{array}\right) \leqslant(k+p)^{p} \leqslant(p+\log x)^{p}
$$

possible values for the string of digits $\left(a_{0}, \ldots, a_{k}\right)$, and therefore for the number of such $n \in \mathcal{A}(x)$. Hence, the total contribution from all $p \leqslant y$ to $\# \mathcal{A}(x)$ is at most

$$
\sum_{p \leqslant y}(p+\log x)^{p} \ll(y+\log x)^{y} \leqslant \exp \left(2 \sqrt{\log x} \log _{2} x\right),
$$

which is negligible when compared with (5.5), and concludes the proof.

5.3. Proof of (ii): divisibility and strict inequality. Since we have established that $\mathfrak{P}_{n+1} \mid \mathfrak{P}_{n}$ for almost all $n \leqslant x$, it is enough to produce a sequence of $n$ of asymptotic density $\ln 2$ such that for each of them there exists a prime $p$ with the property (5.3).

Let $n \leqslant x$ be of the form $n=a p-1$, where $p>\sqrt{x+1}$ and $a \geqslant 2$. Then $a \leqslant(x+1) / p<\sqrt{x+1}<p$.

In particular, $n+1=a p$ with $a<p$, so

$$
s_{p}(n+1)=a<p .
$$


On the other hand, since $n=(a-1) p+p-1$, and $2 \leqslant a<p$, it follows that

$$
s_{p}(n)=a-1+(p-1)=(a-2)+p \geqslant p .
$$

Comparing (5.7) and (5.8), we see that we have (5.3). Obviously, each integer $n \leqslant x$ of this form can be generated by only one prime $p>$ $\sqrt{x+1}$. Fixing $p$, we have that $2 \leqslant a \leqslant(x+1) / p$. Hence, there are $\lfloor(x+1) / p\rfloor-1=x / p+O(1)$ possibilities for $a$. Thus, using the Mertens formula (see [9, Theorem 427]), we see that we generate

$$
\sum_{\sqrt{x+1}<p \leqslant x+1}(x / p+O(1))=x \ln 2+o(x)
$$

such integers $n \leqslant x$ as $x \rightarrow \infty$ in this way, concluding the proof.

5.4. Proof of (iii): equality. We take $n=q-1$ for a prime $q \leqslant x$. Clearly,

$$
s_{q}(n)=q-1<q \quad \text { and } \quad s_{q}(n+1)=1<q .
$$

Thus,

$$
q \nmid \mathfrak{P}_{n} \mathfrak{P}_{n+1} \text {. }
$$

We remark that $q$ is the only prime that divides $n+1$. Thus, Case 2 of Section 5.1 is impossible for any prime $p \neq q$. Hence, the only primes in which $\mathfrak{P}_{n}$ and $\mathfrak{P}_{n+1}$ may differ are the primes $p$ as in Case 1 of Section 5.1 for which $s_{p}(n)=p-1$. In particular, $(p-1) \mid(q-1)$ with $p \neq n$. It is shown in [15] that there exists a positive constant $c$ such that uniformly in $2 \leqslant y \leqslant x$, we have an analogue of (5.4) for shifted primes $q-1$ in place of $n$; that is,

$$
\#\{q \leqslant x:(p-1) \mid(q-1) \text { for some } p \geqslant y\} \ll \frac{x}{\log x(\log y)^{c}},
$$

Again we take $y=\sqrt{\log x}$ and proceeding as in the last part of Section 5.2, in particular, using (5.6) and thus ignoring the fact that $n=q-1$ is a shifted prime in this part of the argument, together with (5.9), we conclude the proof.

\section{ACKNowledgements}

The authors are very grateful to Bernd Kellner and Jonathan Sondow for many useful comments on the initial version of the manuscript.

F.L, P.M. and I.S. would like to thank the Max Planck Institute for Mathematics in Bonn for its hospitality and support during the period when this paper was written. 
The second author was supported in part by NRF (South Africa) Grants CPRR160325161141 and an A-rated researcher award and by CGA (Czech Republic) Grant 17-02804S.

The fourth author was supported in part by ARC (Australia) Grant DP140100118.

\section{REFERENCES}

[1] M. Abramowitz and I. A. Stegun, Handbook of mathematical functions with formulas, graphs, and mathematical tables, 10th printing, with corrections, National Bureau of Standards, A Wiley-Intersci. Pub., New York, 1972. (pp. 3 and 18)

[2] R. Baker and G. Harman, 'The sequence $x / n$ and its subsequences', Rocky Mtn. J. Math., 26 (1996), 795-814. (pp. 5 and 11)

[3] R. C. Baker and G. Harman, 'Small remainder of a vector to suitable modulus', Math. Zeit., 221 (1996), 59-71. (p. 5)

[4] O. Bordellès, Arithmetic tales, Universitext, Springer London, 2012. (p. 13)

[5] Y. Bugeaud, M. Mignotte and S. Siksek, 'Classical and modular approaches to exponential Diophantine equations I. Fibonacci and Lucas perfect powers', Ann. of Math. 163 (2006), 969-1018. (p. 6)

[6] P. Erdős and S. Wagstaff, 'The fractional parts of the Bernoulli numbers', Illinois J. Math. 24 (1980), 104-112. (pp. 5 and 22)

[7] K. Ford, 'Integers divisible by a large shifted prime', Acta Arith., to appear. (pp. 5 and 22)

[8] A. Granville and O. Ramaré, 'Explicit bounds on exponential sums and the scarcity of squarefree binomial coefficients', Mathematika 43 (1996), 73-107. (p. 16)

[9] G. H. Hardy and E. M. Wright, An introduction to the theory of numbers, Oxford Univ. Press, Oxford, 1979. (pp. 2 and 23)

[10] A. Ivić, The Riemann Zeta-Function. Theory and Applications, Dover Pub., Inc., Mineola, New York, 2003. (p. 16)

[11] H. Iwaniec and E. Kowalski, Analytic number theory, Amer. Math. Soc., Providence, RI, 2004. (p. 16)

[12] B. C. Kellner, 'On a product of certain primes', J. Number Theory 179 (2017), 149-164. (pp. 1, 2, 3, and 4)

[13] B. C. Kellner and J. Sondow, 'Power-sum denominators', Amer. Math. Monthly, to appear, (see https://arxiv.org/abs/1705.03857). (pp.1, 2, and 4)

[14] B. C. Kellner and J. Sondow, 'The denominators of power sums of arithmetic progressions', Preprint, 2017, (see https://arxiv.org/abs/1705.05331). (pp. 2 and 5)

[15] F. Luca, A. Pizarro-Madariaga and C. Pomerance, 'On the counting function of irregular primes', Indag. Math. 26 (2015), 147-161. (pp. 5 and 23)

[16] E. M. Matveev, 'An explicit lower bound for a homogeneous rational linear form in the logarithms of algebraic numbers, II', Izv. Ross. Akad. Nauk Ser. Mat. 64 (2000), 125-180; translation in Izv. Math. 64 (2000), 1217-1269. (pp. 5 and 6) 
[17] N. McNew, P. Pollack and C. Pomerance, 'Numbers divisible by a large shifted primes and torsion subgroups of CM ellipitic curves', Int. Math. Res. Notices, to appear. (pp. 5 and 22 )

[18] H. G. Senge and E. G. Straus, 'PV-numbers and sets of multiplicity', Period. Math. Hungar. 3 (1973), 93-100. (p. 6)

[19] C. L. Stewart, 'On the representation of an integer in two different bases', J. Reine Angew. Math. 319 (1980), 63-72. (pp.6 and 7)

[20] J. D. Vaaler, 'Some extremal functions in Fourier analysis', Bull. Amer. Math. Soc., 12 (1985), 183-215. (p. 13)

O.B.: 2 Allée de la COMBe, 43000 Aiguilhe, France

E-mail address: borde430wanadoo.fr

F.L.: School of Mathematics, University of the Witwatersrand, Private Bag X3, Wits 2050, South Africa; Max Planck Institute for Mathematics, Vivatsgasse 7, 53111 Bonn, Germany; Department of Mathematics, Faculty of Sciences, University of Ostrava, 30 Dubna 22, 70103 Ostrava 1, Czech Republic

E-mail address: florian.luca@wits.ac.za

P.M.: Max Planck Institute for Mathematics, Vivatsgasse 7, 53111 Bonn, Germany

E-mail address: moree@mpim-bonn.mpg.de

I.S.: Department of Pure Mathematics, University of New South Wales, 2052 NSW, Australia.

E-mail address: igor.shparlinski@unsw.edu.au 